\title{
Diversity-Multiplexing Tradeoff of the Two-User Interference Channel
}

\author{
Adnan Raja and Pramod Viswanath
}

October 24, 2018

\begin{abstract}
Diversity-Multiplexing tradeoff (DMT) is a coarse high SNR approximation of the fundamental tradeoff between data rate and reliability in a slow fading channel. In this paper, we characterize the fundamental DMT of the two-user single antenna Gaussian interference channel. We show that the class of multilevel superposition coding schemes universally achieves (for all fading statistics) the DMT for the two-user interference channel. For the special case of symmetric DMT, when the two users have identical rate and diversity gain requirements, we characterize the DMT achieved by the Han-Kobayashi scheme, which corresponds to two level superposition coding.
\end{abstract}

\section{Introduction}

Consider the communication scenario depicted in Figure1. Two transmitter-receiver pairs communicate reliably in the face of mutual interference with each other. There is a single antenna at each transmitter and receiver. The discrete time complex baseband model is:

$$
\begin{aligned}
& y_{1}[m]=h_{1} x_{1}[m]+g_{1} x_{2}[m]+z_{1}[m], \\
& y_{2}[m]=h_{2} x_{2}[m]+g_{2} x_{1}[m]+z_{2}[m] .
\end{aligned}
$$

Here $m$ is the time index, $y_{k}$ is the signal at receiver $k$ while $x_{k}$ is the signal sent out by the transmitter $k$ (with $k=1,2$ ). The noise sequences $\left\{z_{1}[m], z_{2}[m]\right\}_{m}$ are i.i.d. complex Gaussian with zero mean and variance $N_{0}$. The transmitters are subject to average power constraints:

$$
\sum_{m=1}^{N}\left|x_{k}[m]\right|^{2} \leq N P_{k}, \quad k=1,2, \quad \forall N \geq 1
$$




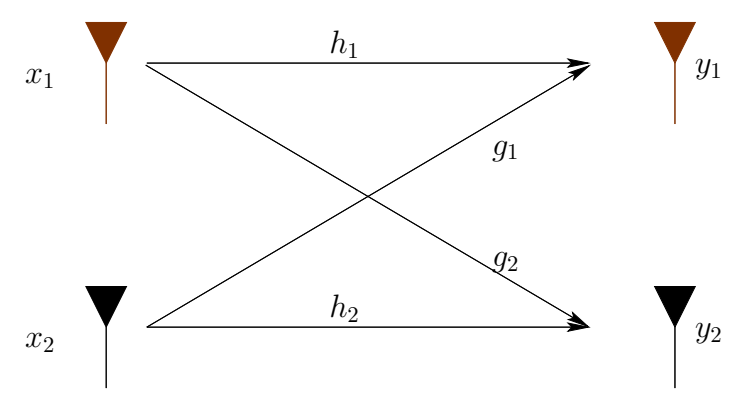

Figure 1: The slow fading two-user Gaussian interference channel.

The complex parameters $\left(h_{1}, g_{1}, h_{2}, g_{2}\right)$ model the channel gain coefficients. Note that $\left(h_{1}, g_{1}\right)$ are the channel gain coefficients corresponding to receiver 1 and $\left(h_{2}, g_{2}\right)$ are the channel gain coefficients corresponding to receiver 2 . A rate pair $\left(R_{1}, R_{2}\right)$ is said to be achievable if transmitter $i$ can send message to receiver $i(i=1,2)$ at rate $R_{i}$ with arbitrarily large reliability. The set of all achievable rate pairs is the capacity region of the channel. In [1], it was shown that there are schemes that achieve within 1 bit per unit time of the capacity of this channel. However, these schemes assume that the channel gain coefficients are known exactly at the transmitters.

The focus of this paper is the slow fading scenario, i.e., the channel gains though random, remain constant over the communication time scale. Correspondingly, we will suppose that the receivers know the channel coefficient gains. In the absence of any feedback link, the transmitters do not know the channel gains; they are aware of the channel statistics, however. This classical setting has received much attention in the context of point to point channels (a classic work is [2], but is also text book material Chapter 9 of [9]) and multiple access channels [3].

Our focus is on the fundamental tradeoff between data rates (for each transmitter) and reliabilities (for each receiver). In the context of slow fading, the fundamental quantity of interest is the outage capacity region: for a given unreliability of communication, this is the pair of rates at which simultaneous reliable communication is possible. Outage capacities are hard to characterize exactly for even the point-to-point channel (formulas have been derived for single antenna channels with specific channel statistics, such as Rayleigh fading). A coarser characterization of this tradeoff is captured by the diversitymultiplexing tradeoff (DMT), first introduced for the point-to-point channel in [2]. The diversity-multiplexing tradeoff is essentially a high SNR approximation, of both the data rate and reliability. The multiplexing gain is defined as

$$
r \stackrel{\text { def }}{=} \lim _{\mathrm{SNR} \rightarrow \infty} \frac{R}{\log \mathrm{SNR}}
$$

The diversity gain is defined as

$$
d \stackrel{\text { def }}{=} \lim _{\mathrm{SNR} \rightarrow \infty}-\frac{\log \mathbb{P}_{\text {out }}}{\log \mathrm{SNR}} .
$$


Stated imprecisely,

$$
\begin{aligned}
R & \approx r \log \mathrm{SNR}, \\
\mathbb{P}_{\text {out }} & \approx \mathrm{SNR}^{-d} .
\end{aligned}
$$

The tradeoff between $d$ and $r$ is the diversity-multiplexing tradeoff.

In the context of the slow fading two-user interference channel, we are interested in finding the largest set of rates of communication $R_{1}$ and $R_{2}$ for the two users while tolerating an outage of $P_{1, \text { out }}$ and $P_{2, \text { out }}$ at the two receivers respectively. In the context of DMT, we want to find the fundamental tradeoff between the multiplexing gains $r_{1}, r_{2}$ and the diversities $d_{1}, d_{2}$ which are defined along the lines of (66) and (7).

Recent work by Akuiyibo and Lévêque [6] has characterized some outer bounds for the DMT of the interference channel. Our results show that these bounds are weak in most cases. In [7] Akçaba and Bölcskei have given an achievable DMT region for the case of very strong interference. Their analysis deals mostly with finite block length schemes.

In this paper, we will allow schemes with arbitrarily large block lengths. Our main result is the characterization of the fundamental DMT for the scalar two-user interference channel. We do this by defining suitable channel outage events for both receivers. When in outage, the receivers would very likely be in error. However, when the channel is not in outage, the compound channel result of [5] ensures that a multilevel superposition coding scheme with opportunistic decoding of interference achieves the required rate. Hence, we show that this scheme achieves the DMT universally (for all channel statistics).

The Han-Kobayashi scheme is a special case (when the number of levels are restricted to two) of the more general multi-level superposition coding scheme introduced in [5]. We characterize the performance of the Han-Kobayashi scheme in the context of the special case of symmetric DMT, where the two users have identical rate and diversity requirements. We compare this symmetric DMT achievable scheme by this scheme, to an outer-bound obtained by considering the worst case channel outer bound of the compound interference channel. While the Han-kobayashi scheme is DMT-optimal in some instances, we also see that it is not DMT-optimal in many other cases.

The rest of this paper is organized as follows. In Section 2 we quickly describe the system model and assumptions. We characterize the fundamental DMT of the two-user interference channel by relating it to the compound interference channel problem in Section 3. In Section 4 we characterize the performance of the Han-Kobayashi scheme for the case of symmetric DMT, where the rate and diversity requirements for the two users are identical. In Section 5 we consider two important special cases - the Z-interference channel and the interference channel with only the cross links fading - for which the performance of the Han-Kobayashi scheme is DMT optimal.

Notation: We will use $(a \vee b)$ to denote the maximum of $a$ and $b$, and $(a \wedge b)$ to denote the minimum of $a$ and $b .(x)^{+}$will be used to denote $(x \vee 0) . f(x) \doteq g(x)$ will be used to 
denote

$$
\lim _{x \rightarrow \infty} \frac{\log f(x)}{\log g(x)}=1
$$

The symbols $\dot{<}, \dot{>}, \dot{\leq}$ and $\geq$ will be used in the same spirit. $\mathbb{P}($.$) will be used to denote$ the probability of an event.

\section{Model}

The two-user interference channel model described in (11) and (21) can be written in the following equivalent form.

$$
\begin{aligned}
& \tilde{y}_{1}[m]=h_{1} \sqrt{\mathrm{SNR}_{1}} \tilde{x}_{1}[m]+g_{1} \sqrt{\mathrm{INR}_{1}} \tilde{x}_{2}[m]+\tilde{z}_{1}[m], \\
& \tilde{y}_{2}[m]=h_{2} \sqrt{\mathrm{SNR}_{2}} \tilde{x}_{2}[m]+g_{2} \sqrt{\mathrm{INR}_{2}} \tilde{x}_{1}[m]+\tilde{z}_{2}[m] .
\end{aligned}
$$

Note that $\mathrm{SNR}_{k}$ and $\mathrm{INR}_{k}$ are the average signal-to-noise and interference-to-noise ratios at receiver $k$ respectively $(k=1,2)$. The normalized i.i.d. noise sequences $\left\{\tilde{z}_{1}[m], \tilde{z}_{2}[m]\right\}_{m}$ have unit variance and the normalized transmit sequences $\left\{\tilde{x}_{1}[m], \tilde{x}_{2}[m]\right\}_{m}$ are subject to a unit average power constraint.

We will assume that the channel gain coefficients, $\left\{h_{1}, g_{1}, h_{2}, g_{2}\right\}$, are also normalized and are zero mean and unit variance random variables. We will not suppose any particular statistical distribution for the channel gains. We will only assume that the channel gains exhibit an exponential tail, i.e., for $X \in\left\{h_{1}, g_{1}, h_{2}, g_{2}\right\}$ there exists a $\gamma>0$ such that

$$
\lim _{x \rightarrow \infty} \frac{\mathbb{P}\left(|X|^{2} \geq x\right)}{e^{-\gamma x}} \leq 1
$$

The DMT will be characterized by the near-zero behavior of the distribution functions, which is characterized by $\kappa_{X}$ for $X \in\left\{h_{1}, g_{1}, h_{2}, g_{2}\right\}$ and is given by,

$$
\kappa_{X} \stackrel{\text { def }}{=} \lim _{\epsilon \rightarrow 0+} \frac{\log \mathbb{P}\left(|X|^{2}<\epsilon\right)}{\log \epsilon} .
$$

Note that $\kappa_{X} \geq 0$.

We also introduce the following relative scaling parameters for the average signal-to noise-ratio, $\mathrm{SNR}_{i}$, and interference-to-noise ratio, $\mathrm{INR}_{i}$, at the receivers, along the lines of [1].

$$
\begin{gathered}
\beta_{i} \stackrel{\text { def }}{=} \frac{\log \mathrm{SNR}_{i}}{\log \mathrm{SNR}}, \\
\alpha_{i} \stackrel{\text { def }}{=} \frac{\log \mathrm{INR}_{i}}{\log \mathrm{SNR}} .
\end{gathered}
$$


Here $\mathrm{SNR}=P_{1} / N_{0}$. The parameters $\left\{\beta_{1}, \alpha_{1}, \beta_{2}, \alpha_{2}\right\}$ are the relative strength level of the direct and cross links in $\mathrm{dB}$ scale. We will study the scaling behavior of the rate and reliability with SNR keeping these parameters fixed.

We say that a diversity-multiplexing point $\left(d_{1}, d_{2}, r_{1}, r_{2}\right)$ is achievable for the two-user interference channel with parameters $\left\{\beta_{1}, \alpha_{1}, \beta_{2}, \alpha_{2}\right\}$ if there exists a sequence of schemes, one for each (integer-valued, say) SNR, with rate pair $\left(R_{1}, R_{2}\right)$ given by

$$
R_{1}=r_{1} \log \mathrm{SNR} \quad \text { and } \quad R_{2}=r_{2} \log \mathrm{SNR},
$$

and probability of error for the two receivers $\mathbb{P}\left(\mathcal{E}_{1}\right)$ and $\mathbb{P}\left(\mathcal{E}_{2}\right)$ respectively, satisfying

$$
\mathbb{P}\left(\mathcal{E}_{1}\right) \leq \mathrm{SNR}^{-d_{1}} \quad \text { and } \quad \mathbb{P}\left(\mathcal{E}_{1}\right) \leq \mathrm{SNR}^{-d_{2}}
$$

The set of all achievable diversity-multiplexing points constitute the fundamental diversitymultiplexing tradeoff region. By $\mathcal{R}\left(d_{1}, d_{2}\right)$, we will denote the fundamental multiplexing gain region for given diversity gains $d_{1}$ and $d_{2}$. To characterize the DMT region, our approach will be to characterize $\mathcal{R}\left(d_{1}, d_{2}\right)$ for every diversity gain pair $\left(d_{1}, d_{2}\right)$.

\section{Fundamental DMT Region}

The outage formulation provides a natural approach to build fundamental limits on the DMT. In this section, we will characterize the fundamental DMT for the two-user slow fading interference channel by relating it to the compound interference channel defined over an appropriate no-outage set.

For given diversity gain requirements $d_{1}$ and $d_{2}$ for the two users, we characterize the fundamental multiplexing gain region $\mathcal{R}\left(d_{1}, d_{2}\right)$. We do this by defining outage events $O_{1}$ and $\mathrm{O}_{2}$ for the two users with probability of the order $\mathrm{SNR}^{-d_{1}}$ and $\mathrm{SNR}^{-d_{2}}$ respectively. These outage events are defined so that they are largest in the scale of interest. Then, we use the compound channel result of [5] to characterize the generalized degree of freedom region of the compound interference channel defined by the no-outage set, denoted by $\mathcal{C}\left(d_{1}, d_{2}\right)$.

The main result of this paper is that the generalized degree of freedom of the compound interference channel is the fundamental multiplexing region of the interference channel. Further, the achievability theorem for the compound interference channel in [5] ensures that the generalized multi-level superposition coding scheme with opportunistic decoding of interference achieves the fundamental DMT of the interference channel. 


\subsection{Outage Formulation}

We introduce the following change of variables as is done in [2].

$$
\hat{X} \stackrel{\text { def }}{=}-\frac{\log \left(|X|^{2}\right)}{\log \mathrm{SNR}}
$$

for $X \in\left\{h_{1}, g_{1}, h_{2}, g_{2}\right\}$. $\hat{X}$ is in a way the measure of the order of the magnitude of the channel gain with respect to SNR.

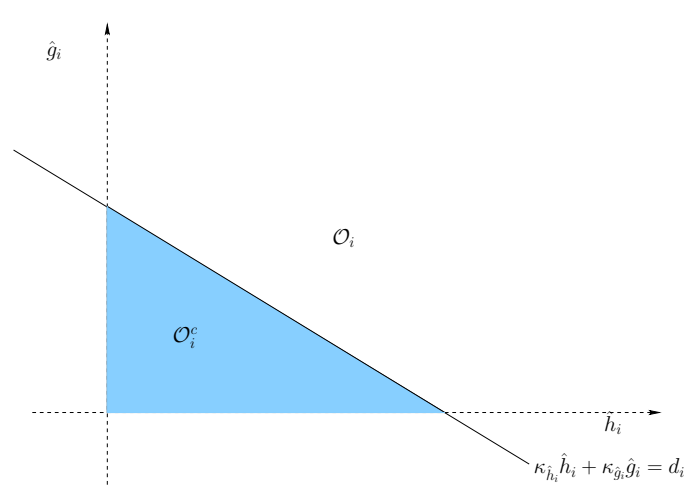

Figure 2: Partition of $\left(\hat{h}_{i}, \hat{g}_{i}\right)$-space into outage and no-outage sets

We now define the complement of the outage events for the two receivers, i.e. $\hat{\mathcal{O}}_{1}^{c}$ and $\hat{\mathcal{O}}_{2}^{c}$ respectively, in terms of the order random variables $\hat{X}$ 's. The outage and the no-outage sets are pictorially depicted in Figure 2]

$$
\hat{\mathcal{O}}_{i}^{c} \stackrel{\text { def }}{=}\left\{\left(\hat{h}_{i}, \hat{g}_{i}\right): \kappa_{h_{i}} \hat{h}_{i}+\kappa_{g_{i}} \hat{g}_{i}<d_{i}, \hat{h}_{i}, \hat{g}_{i} \geq 0\right\} .
$$

The outage events so defined satisfy two important properties as stated in the following Lemma.

Lemma 1. (i) If $E_{i}^{\epsilon} \subset \hat{\mathcal{O}}_{i}^{c}$ is an infinitesimal small square element given by,

$$
E_{i}^{\epsilon}=\left[h^{\prime}, h^{\prime}+\epsilon\right) \times\left[g^{\prime}, g^{\prime}+\epsilon\right),
$$

then

$$
\mathbb{P}\left(E_{i}^{\epsilon}\right) \geq \mathrm{SNR}^{-d_{i}+\delta}
$$

for some $\delta>0$.

(ii) $\mathbb{P}\left(\hat{\mathcal{O}}_{i}\right) \doteq \mathrm{SNR}^{-d_{i}}, \quad i=1,2$.

Proof: The reader is referred to Appendix $\AA$ for the details of the proof. The result follows from the properties (11) and (12) assumed for the distribution functions. In particular part (ii) follows from Laplace's method as in [2] and [4. 
We now consider the compound interference channel defined by the set of channel states $\hat{\mathcal{A}}$, where

$$
\hat{\mathcal{A}}=\hat{\mathcal{O}}_{1}^{c} \times \hat{\mathcal{O}}_{2}^{c} .
$$

We denote this by $\mathcal{I}(\hat{\mathcal{A}})$. Note that since $\hat{\mathcal{A}}$ is a function of SNR, $\mathcal{I}$ is a family of compound interference channels indexed by SNR, with fixed parameters $\left\{\beta_{1}, \alpha_{1}, \beta_{2}, \alpha_{2}\right\}$ and $\left(d_{1}, d_{2}\right)$.

Let $\mathcal{C}(\hat{\mathcal{A}})$ denote the capacity region of the compound interference channel $\mathcal{I}(\hat{\mathcal{A}})$. We define the generalized degree of freedom (g.d.o.f.) for this family of compound interference channel $\mathcal{I}$ as

$$
\mathcal{C}\left(d_{1}, d_{2}\right) \stackrel{\text { def }}{=} \limsup _{\text {SNR } \rightarrow \infty} \frac{\mathcal{C}(\hat{\mathcal{A}})}{\log \mathrm{SNR}} .
$$

Note that this is an extension of the definition of the g.d.o.f. of a family of interference channels in [1] to the case of a family of compound interference channels.

\subsection{Main Result}

The main result of this paper is the following theorem which relates the fundamental multiplexing region of the two-user interference channel $\mathcal{R}\left(d_{1}, d_{2}\right)$ defined in section 2 to the g.d.o.f. defined of the family of compound of interference channel $\mathcal{C}\left(d_{1}, d_{2}\right)$ defined in the previous subsection.

\section{Theorem 1.}

$$
\mathcal{R}\left(d_{1}, d_{2}\right)=\mathcal{C}\left(d_{1}, d_{2}\right)
$$

Proof:

We will first show that $\mathcal{C}\left(d_{1}, d_{2}\right) \subseteq \mathcal{R}\left(d_{1}, d_{2}\right)$. Consider $\left(r_{1}, r_{2}\right) \in \mathcal{C}\left(d_{1}, d_{2}\right)$. This implies that there is a family of schemes, one for each SNR, that achieves the rate pair $\left(r_{1} \log \mathrm{SNR}, r_{2} \log \mathrm{SNR}\right)$ for the compound interference channel $\mathcal{I}(\hat{\mathcal{A}})$. For these schemes the probability of error at receiver $i=1,2$, can be bounded as

$$
\mathbb{P}\left(\mathcal{E}_{i}\right)=\mathbb{P}\left(\mathcal{O}_{i}\right) \mathbb{P}\left(\mathcal{E}_{i} \mid \mathcal{O}_{i}\right)+\mathbb{P}\left(\mathcal{O}_{i}^{c}\right) \mathbb{P}\left(\mathcal{E}_{i} \mid \mathcal{O}_{i}^{c}\right) .
$$

Since $\left(r_{1}, r_{2}\right) \in \mathcal{C}\left(d_{1}, d_{2}\right)$, we have $\mathbb{P}\left(\mathcal{E}_{i} \mid \mathcal{O}_{i}^{c}\right) \rightarrow 0$. Thus, we can bound the probability of error as

$$
\mathbb{P}\left(\mathcal{E}_{i}\right) \leq \mathbb{P}\left(\mathcal{O}_{i}\right) \doteq \mathrm{SNR}^{-d_{i}} .
$$

Therefore, $\left(r_{1}, r_{2}\right) \in \mathcal{R}\left(d_{1}, d_{2}\right)$.

Next we will show that $\mathcal{R}\left(d_{1}, d_{2}\right) \subseteq \mathcal{C}\left(d_{1}, d_{2}\right)$ Consider $\left(r_{1}, r_{2}\right) \in \mathcal{R}\left(d_{1}, d_{2}\right)$. There is a family of schemes, one for each SNR, with rate pairs $\left(r_{1} \log \mathrm{SNR}, r_{2} \log \mathrm{SNR}\right)$ and with probability of error at the two receivers, $\mathbb{P}\left(\mathcal{E}_{i}\right), i=1,2$ satisfying

$$
\mathbb{P}\left(\mathcal{E}_{i}\right) \leq \mathrm{SNR}^{-d_{i}} .
$$


Let $E_{i}^{\epsilon}$ denote any infinitesimally small subset of $\hat{\mathcal{O}}_{i}^{c}$ as defined in (19). From Lemma 1, we know that

$$
\mathbb{P}\left(E_{i}^{\epsilon}\right) \geq \mathrm{SNR}^{-d_{i}+\delta}
$$

We can lower bound the probability of error as follows

$$
\mathbb{P}\left(\mathcal{E}_{i}\right) \geq \mathbb{P}\left(\mathcal{E}_{i} \cap E_{i}^{\epsilon}\right)=\mathbb{P}\left(E_{i}^{\epsilon}\right) \mathbb{P}\left(\mathcal{E}_{i} \mid E_{i}^{\epsilon}\right) .
$$

Therefore

$$
\mathbb{P}\left(\mathcal{E}_{i} \mid E_{i}^{\epsilon}\right) \leq \frac{\mathbb{P}\left(\mathcal{E}_{i}\right)}{\mathbb{P}\left(E_{i}^{\epsilon}\right)} \leq \mathrm{SNR}^{-\delta} .
$$

As $\mathrm{SNR} \rightarrow \infty, P\left(\mathcal{E}_{i} \mid E_{i}\right) \rightarrow 0$. Therefore, $\left(r_{1}, r_{2}\right) \in \mathcal{C}\left(d_{1}, d_{2}\right)$. And hence $\mathcal{R}\left(d_{1}, d_{2}\right) \subseteq$ $\mathcal{C}\left(d_{1}, d_{2}\right)$.

\subsection{Achievability of the Fundamental DMT Region}

In [5], a coding theorem for the compound two-user Gaussian interference channel was given. The scheme used was a multi-level superposition coding scheme with opportunistic decoding of interference. This scheme is a generalization of the two-level superposition coding scheme, which is also called the Han-Kobayashi scheme. From theorem 1 and the coding theorem in [5] the following result follows.

Theorem 2. The multi-level superposition coding scheme with opportunistic decoding of interference is approximately universal for the slow-fading interference channel, i.e. it achieves the fundamental DMT region of the scalar two-user interference channel.

Proof: It was shown in [5] that multi-level superposition coding scheme achieves within $1 \mathrm{bit} / \mathrm{symbol}$ of the capacity region of the finite state compound interference channel. The finite state assumption required that the set of states defining the compound channel have finite cardinality. If the number of states is $N$, then the scheme essentially has $N+1$ independent data streams superposed together. Depending on the channel state, a suitable number of streams from the interfering user is decoded. In our problem, the set $\mathcal{A}$ is a continuum of states and hence the compound interference channel defined by the set $\mathcal{A}$ has infinite states.

The compound interference channel result of [5] for the finite state can be easily extended to the infinite state by doing a finite quantization of the infinite state set. We can then use a finite-level superposition coding scheme corresponding to the quantized finite state compound channel. We can get as close as desired to within $1 \mathrm{bit} / \mathrm{symbol}$ of the capacity region by taking a fine enough quantization.

In the limiting case, as SNR goes to infinity, the constant 1 bit/symbol gap disappears and the superposition coding scheme achieves the g.d.o.f. $\mathcal{C}\left(d_{1}, d_{2}\right)$ and due to Theorem 1 achieves $\mathcal{R}\left(d_{1}, d_{2}\right)$. 


\section{Han-Kobayashi Scheme and Symmetric DMT}

In the previous section we showed that the multi-level superposition coding scheme with opportunistic decoding of interference achieves the DMT of the two-user interference channel, universally for all fading channel statistics. However, an explicit characterization of the DMT region is elusive. This is due to the complexity in explicitly finding the g.d.o.f. region for the compound interference channel achieved by the multi-level superposition coding scheme as seen in [5].

The two-level version of this scheme is the familiar Han-Kobayashi scheme. It is an interesting question to ask as to how far from optimal is this scheme with respect to the DMT. In Section 4.1, we answer this question for the symmetric case, where we impose identical rate and diversity requirements for both the users. It turns out that the HanKobayashi scheme is DMT optimal in many regimes. We show this by comparing its performance to an outer bound derived from considering the worst case outer bound of the corresponding compound interference channel in Section 4.2. However, in Section 4.3 we show with an example that both the Han-Kobayashi scheme and the worst case outer bound are not tight for all regimes.

\subsection{DMT Achieved by the Han-Kobayashi Scheme}

The focus of this section is on the DMT performance of the Han-Kobayashi scheme. We analyze its performance explicitly, but in the specific context below:

- We suppose the statistics of the channel gains to be symmetric with respect to the two users i.e.,

$$
\beta_{1}=\beta_{2}=1, \quad \alpha_{1}=\alpha_{2}=\alpha \text {. }
$$

- We suppose that the near-zero behavior of the distribution function to be linear (example: Rayleigh distribution), i.e. $\kappa_{X}=1$, for $X=\left\{h_{1}, g_{1}, h_{2}, g_{2}\right\}$. This implies that

$$
\mathbb{P}\left(\frac{|X|^{2}}{E\left[|X|^{2}\right]}<\epsilon\right) \approx \epsilon,
$$

for small $\epsilon>0$.

- We only consider symmetric diversity and rate requirements for the two receivers, i.e.,

$$
d_{1}=d_{2}=d, \quad r_{1}=r_{2}=r
$$


The Han-Kobayashi scheme is a two-level superposition scheme and achieves the generalized degree of freedom region of the interference channel [1]. The scheme can be succinctly described as the following. Each user splits its message into two parts - public and private - and superposition-codes them. The public is decoded by both the receivers, while the private is decoded only by the intended receiver. Gaussian code books with rate $s_{i} \log \mathrm{SNR}$ and $t_{i} \log \mathrm{SNR}$ are used for both the private and public messages respectively. The actual codeword transmitted is the sum of the public and private codewords. Therefore, the rate achieved for each user is $r_{i} \log \mathrm{SNR}$, where

$$
r_{i}=s_{i}+t_{i}
$$

An important parameter of this scheme is the power split between the public and private streams. Let $\rho_{v_{i}}$ denote the fraction of power allocated to the private of user $i$. Since we are concerned with high SNR approximations, we will let

$$
\rho_{v_{i}} \doteq \mathrm{SNR}^{-v_{i}}
$$

where $v_{i}>0$. The fraction of power allocated to the public will be given by

$$
\begin{aligned}
\rho_{u_{i}} & =1-\rho_{v_{i}} \\
& \doteq 1-\mathrm{SNR}^{-v_{i}} \\
& \doteq 1
\end{aligned}
$$

The parameter $v_{i}$, therefore, determines the power split for user $i$.

Consider the case when there is no fading; so, the channel gains are the mean values, i.e. unity. The interference level (in the relative $\mathrm{dB}$ scale) is $\alpha$. In [1] it was shown that a good choice for the power split is such that the private stream appears at noise level at the interfering receiver i.e.,

$$
\begin{array}{cl}
\rho_{v_{i}} \mathrm{SNR}^{\alpha} & =1 \\
v_{i} & =\alpha .
\end{array}
$$

Suppose the channel cross gains $\left|g_{i}\right|^{2}$ take values $\mathrm{SNR}^{-\hat{g}_{i}}$, for $i=1,2$. Then the effective interference level for the two links are $\alpha-\hat{g}_{i}, i=1,2$. Correspondingly, the power split for the two users is given by $\alpha-\hat{g}_{2}$ and $\alpha-\hat{g}_{1}$, respectively.

It is interesting to note that the power split does not depend on the level of the direct links. The direct links only limit the rates. For our compound channel problem, as the cross gain takes values in the no-outage set, the interference level takes values in the range $(\alpha-d, \alpha)$ for each link independently. We need to find a fixed scheme with optimal powersplit and suitable rates for the public and private messages, that works for the compound channel. This is a routine optimization problem and the results of this calculation (done in Appendix (B) are summarized in Table[1. Figure 3 illustrates the optimal tradeoff curve that can be achieved by the Han-Kobayashi scheme for different values of $\alpha$. 
Table 1: DMT achieved by the Han-Kobayashi scheme

\begin{tabular}{|c|c|c|c|}
\hline$\alpha$ & $d$ & $v$ & $r_{\mathrm{HK}}(\alpha, d)$ \\
\hline \hline$\alpha \geq 1$ & $1 \geq d \geq 0$ & (All public) & $(1-d) \wedge \frac{1-d+\alpha}{4}$ \\
\hline $1>\alpha \geq 0$ & $1 \geq d>1-\alpha$ & $($ All public) & $(1-d) \wedge \frac{1-d+\alpha}{4}$ \\
\hline $1>\alpha \geq \frac{2}{3}$ & $1-\alpha \geq d \geq 0$ & $\alpha$ & $1-\frac{\alpha}{2}-d$ \\
\hline$\frac{2}{3}>\alpha \geq \frac{5}{8}$ & $1-\alpha \geq d>5 \alpha-3$ & $\frac{3 \alpha+d-1}{2}$ & $\frac{3-\alpha-3 d}{4}$ \\
& $5 \alpha-3 \geq d \geq 0$ & $\alpha$ & $(\alpha-d)$ \\
\hline$\frac{5}{8}>\alpha \geq 0$ & $1-\alpha \geq d>1-\frac{7 \alpha}{5}$ & $\frac{3 \alpha+d-1}{2}$ & $\frac{3-\alpha-3 d}{4}$ \\
& $1-\frac{7 \alpha}{5} \geq d>\left(\alpha-\frac{1}{2}\right) \vee(1-2 \alpha)$ & $\frac{1+\alpha-d}{3}$ & $\frac{1+\alpha-d}{3}$ \\
& $\left(\alpha-\frac{1}{2}\right) \vee(1-2 \alpha) \geq d \geq 0$ & $\alpha$ & $(\alpha-d) \vee(1-\alpha-d)$ \\
\hline
\end{tabular}

\subsection{Comparison to the Worst Case Outer Bound}

To evaluate the performance of the Han-Kobayashi scheme we use a simple outer bound to the DMT, obtained by using the worst case outer bound of the compound channel. This is equivalent to supposing that the transmitter also has channel information. We know that the capacity of the compound channel cannot be larger than the capacity of the worst channel in the set, i.e.,

$$
C(\hat{\mathcal{A}}) \leq \min _{\hat{a} \in \hat{\mathcal{A}}} C(\hat{a}) .
$$

Here $C(\hat{\mathcal{A}})$ is the symmetric d.o.f. of the compound channel and $C(\hat{a})$ is the symmetric d.o.f. of the two-user interference channel with channel coefficients $\hat{a}=\left(\hat{h}_{1}, \hat{g}_{1}, \hat{h}_{2}, \hat{g}_{2}\right)$ and can be easily computed [1]. The detailed analysis can be found in Appendix []. The worst case outer bound is also plotted for different values of $\alpha$ in Fig. 3

Note that when the diversity gain needed is larger, we need a coding scheme that is resilient to a larger range of fading. We can summarize our understanding by breaking the diversity gain into three regimes.

- Large diversity regime: When the diversity gain is large enough $\left(d>(1-\alpha)^{+}\right)$, the dominant error event is the direct link fading so much, so as to drive the interference channel into the strong and very strong interference regime. Therefore decoding the complete message from both the users at both the receivers is DMT optimal in this regime.

- Small diversity regime: We define this to be the regime where the Han-Kobayashi scheme with the power split corresponding to the mean level of interference $(v=\alpha)$ is DMT optimal. This is true when the diversity gain is small enough (which depends on $\alpha$ as seen from Fig. 3 and Table 1). Since the fading range over which our coding scheme must work is small, the perturbation in the strength of the channel links from the mean level is small and a scheme designed for the mean level still remains optimal in the DMT sense. 
- Intermediate diversity regime: This is the regime which is complement to the first two regimes. Our optimization suggests what power-split level to choose for the HanKobayashi scheme. While this choice is DMT optimal for some part of the regime, in general it is not. This is due to the non-monotonic behavior of the symmetric rate versus the cross link strength as seen from the well known 'W-shaped' curve in [1].

\subsection{Improving the HK scheme and the Outer Bound}

The previous section shows that the Han-Kobayashi scheme does not meet the worst case outer bound in many regimes. In the rest of this section, we see that both the inner and outer bounds can be improved. We will do this by considering the specific case when

$$
\begin{aligned}
\alpha & =1 / 2 \\
d & =1-5 \alpha / 4=3 / 8 .
\end{aligned}
$$

From Figure 3d, we see that

$$
r_{\mathrm{HK}}(1 / 2,3 / 8)=11 / 32=0.34375,
$$

whereas

$$
r_{\text {out }}(1 / 2,3 / 8)=3 / 8=0.375 .
$$

\subsubsection{Beyond Han-Kobayashi Scheme}

In [5], we generalized the Han-Kobayashi scheme to a multi-level superposition coding scheme. In particular, we will now consider a three-level superposition coding scheme. Accordingly, each user splits its message into three parts with power splits given by,

$$
\rho_{v_{i 1}} \doteq \mathrm{SNR}^{-v_{1}}, \quad \rho_{v_{i 2}} \doteq \mathrm{SNR}^{-v_{2}} \text {, and } \quad \rho_{v_{i 3}} \doteq 1, \quad \text { for } i=1,2 .
$$

The receiver decodes one or two messages from the interfering user depending on the interference level. Accordingly, we will define a threshold $\gamma$ such that, when $\hat{g}_{i} \geq \gamma$ the receiver decodes two messages, and when $\hat{g}_{i}<\gamma$ the receiver decodes one message. The resulting expressions describing the rates achievable by this scheme are prohibitively complex to describe. And hence, we will just describe our results. Optimizing the values for $v_{1}, v_{2}$ and $\gamma$ to achieve the maximum symmetric rate gives,

$$
r(1 / 2,3 / 8)=55 / 152=0.361842105,
$$

when,

$$
v_{1}=73 / 152, \quad v_{2}=49 / 152, \text { and } \quad \gamma=56 / 152 .
$$




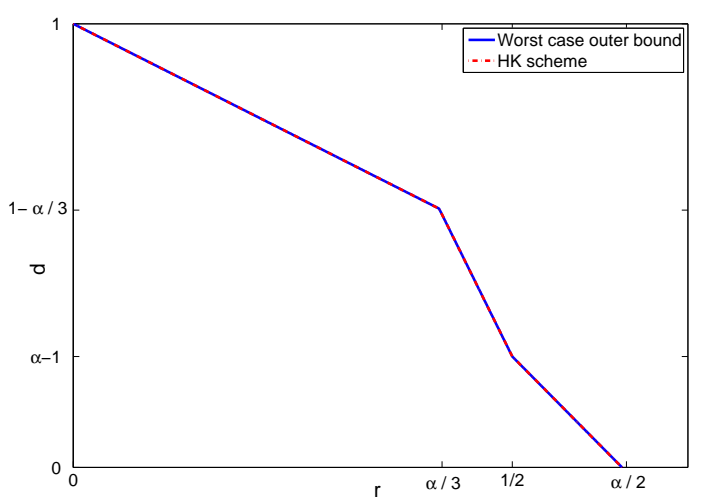

(a) $1 \leq \alpha$

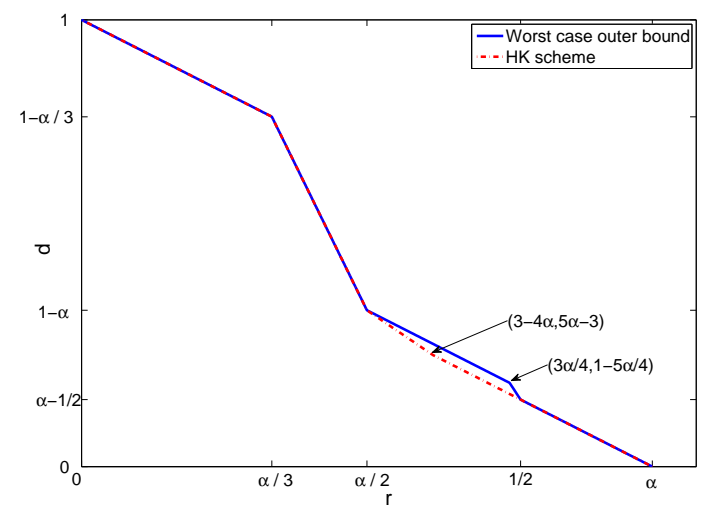

(c) $5 / 8 \leq \alpha \leq 2 / 3$

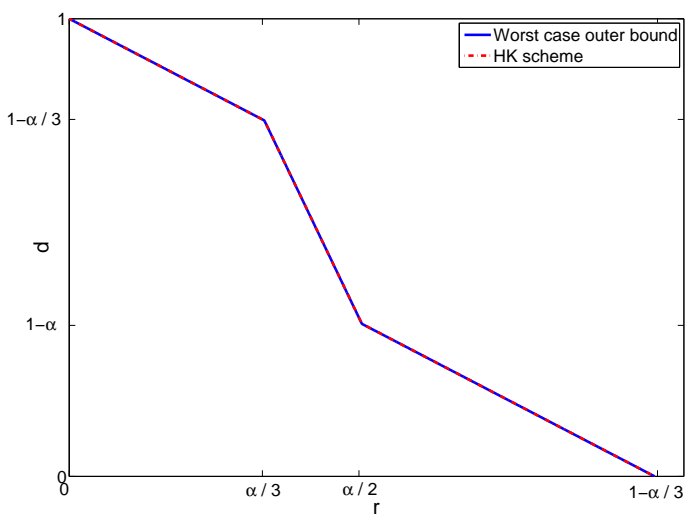

(b) $2 / 3 \leq \alpha \leq 1$

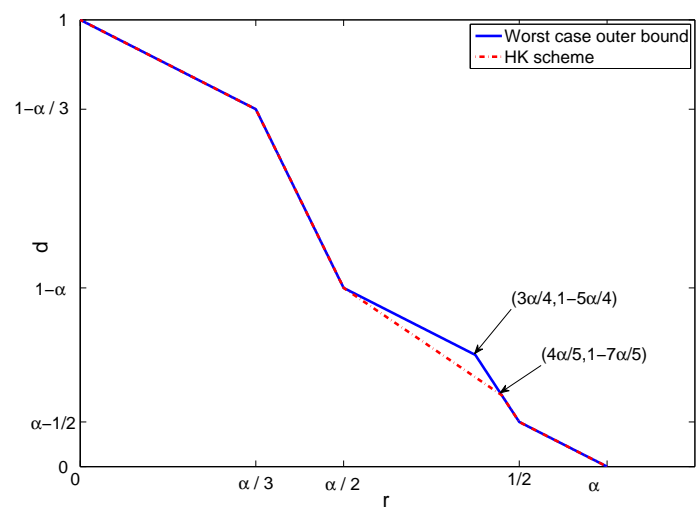

(d) $1 / 2 \leq \alpha \leq 5 / 8$

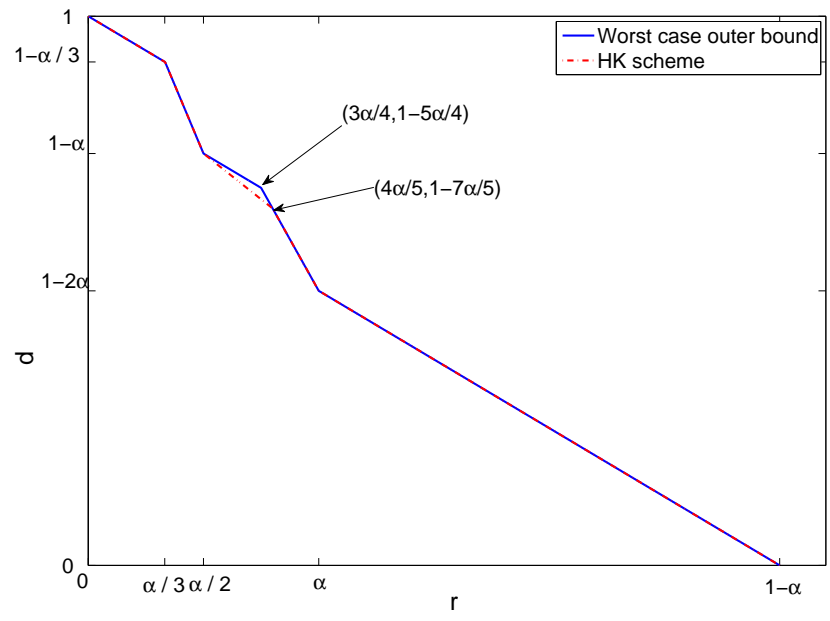

(e) $0 \leq \alpha \leq 1 / 2$

Figure 3: Symmetric DMT 


\subsubsection{Beyond the worst-case outer bound}

We can obtain a better outer bound to the DMT by obtaining a better outer bound to the capacity of the compound channel described by the $\hat{\mathcal{A}}$. We can do this by taking a finite-

state quantization of the set $\hat{\mathcal{A}}$, say $\hat{\mathcal{A}}_{Q}$. In [5], we described the capacity of the finite state compound interference channel. Note that this capacity region was obtained as a two-dimensional projection of many higher dimensional polytopes and was complicated to describe. However, numerically, given fixed values of the states, the symmetric capacity can be obtained easily by a linear program.

In our analysis, we used a uniform quantization given by

$$
\hat{g}_{i k}=\alpha-\frac{k d}{Q-1}, \text { and } \quad \hat{h}_{i k}=d-\hat{g}_{i k}, \quad 0 \leq k \leq Q-1, i=1,2
$$

For $Q=16$, we can get

$$
r_{\text {out }}(1 / 2,3 / 8)=0.3667
$$

\section{Important Instances}

In our discussion so far we have considered all four links in the interference channel to be potentially faded. In this section, we consider two importance instances of the interference channel which do not share this characteristic. In Section 5.1 we will consider the case of Zinterference channel, where only one of the user is interfering with the other. In Section 5.2 we will consider the case where only the cross links are fading and the direct links are fixed. Both these cases share an interesting result: we can have a closed form characterization of the optimal DMT and, furthermore, the Han-Kobayashi scheme achieves it.

\section{$5.1 \quad$ Z-Interference Channel}

The Z-Interference channel is illustrated in Figure4. The figure shows the average relative strength of the links $\beta_{1}, \beta_{2}$ and $\alpha_{1}$ as given by (13) and (14). Note that for the ZInterference channel the channel gain coefficient $g_{2}$ is zero; this implies that $\alpha_{2}=-\infty$. For simplicity of analysis, we will assume the near-zero behavior of the distribution function to be linear, i.e. $\kappa_{X}=1$ for $X=\left\{h_{1}, g_{1}, h_{2}\right\}$ (example: Rayleigh distribution).

Theorem 3. The fundamental multiplexing gain region $\mathcal{R}(d, d)$ for the Raleigh fading 


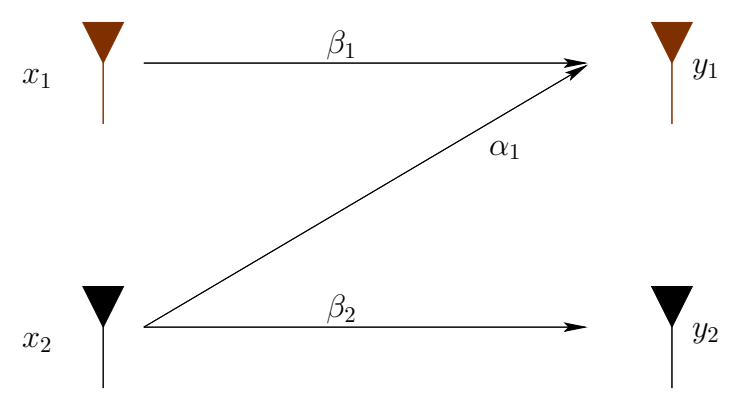

Figure 4: The slow fading two-user Gaussian Z-interference channel.

two-user Gaussian Z-interference channel with $\beta_{1}=\beta_{2}=1$ and $\alpha_{1}=\alpha$ is given by

$$
\begin{aligned}
\mathcal{R}(d, d)=\left\{r_{1}\right. & \leq(1-d)^{+} \\
r_{2} & \leq(1-d)^{+} \\
r_{1}+r_{2} & \left.\leq\left(1-d \vee \alpha-d \vee \frac{1-d+\alpha}{2}\right)+(1-\alpha-d)^{+}\right\} .
\end{aligned}
$$

Further, a simple Han-Kobayashi scheme achieves this fundamental DMT region.

Proof: The details of the proof are in Appendix D, The idea is to characterize the DMT region achievable by a simple Han-Kobayashi scheme with power split $v_{1}=0$ and $v_{2}=\alpha_{1}$. It turns out that this meets the simple worst case outer bound.

\section{$5.2 \quad$ Fading Interfering Links}

In many practical settings, a wireless link (between a single source and destination) is power controlled so as to effectively have no effect of fading. We turn to such a scenario now: the direct link gains $h_{1}$ and $h_{2}$ are fixed, while the interfering links are susceptible to channel fading. This is equivalent to assuming that

$$
\kappa_{h_{i}}=0, \quad i=1,2 .
$$

For simplicity, we will also assume the average relative strength of the links is symmetric and is given by

$$
\beta_{1}=\beta_{2}=1, \quad \alpha_{1}=\alpha_{2}=\alpha .
$$

As usual we suppose that the near-zero behavior of the distribution function of the cross gains $X=\left\{g_{1}, g_{2}\right\}$ to be linear, i.e., $\kappa_{X}=1$ (example: Rayleigh distribution).

Theorem 4. The symmetric DMT of the interference channel with only cross links fading is given by

$$
r(d)=1 \wedge\left(\frac{1}{2} \vee 1-\alpha \vee \alpha-d\right) \wedge\left(\frac{1}{2} \vee 1-\frac{\alpha}{2} \vee \frac{\alpha-d}{2}\right) .
$$


Proof: The no-outage set is now given by

$$
\hat{\mathcal{A}}=\left\{0 \leq \hat{g}_{1} \leq d_{1}\right\} \times\left\{0 \leq \hat{g}_{2} \leq d_{2}\right\} .
$$

We obtain the worst case outer bound by following the analysis similar to that in Appendix C and using (91) (Note that for our current setup $\hat{h}_{1}=\hat{h}_{2}=0$ ). It can be shown that the worst case outer bound is (50).

Turning to achievable schemes: we first note that the simple scheme of orthogonalizing the two users, so that they don't interfere with each other, achieves a multiplexing gain of $1 / 2$ to support arbitrary diversity. This is possible because in this model the direct links are not fading. This scheme meets the outer-bound for $d \geq \alpha-1$, when $\alpha>1$, and for $d \geq \alpha-1 / 2$ when $\alpha \leq 1$.

Another simple scheme is to treat interference as noise and it can be shown that for $\alpha \leq 1 / 2$, it meets the optimal multiplexing gain of $1-\alpha$ for arbitrary diversity gains.

For the rest of the cases, we can characterize the symmetric DMT achieved by the Han-Kobayashi scheme with power splits given by $v_{1}=v_{2}=\alpha$. Using (80), we can show that this scheme achieves the symmetric DMT given by

$$
r(d)=1 \wedge(1-\alpha \vee \alpha-d) \wedge\left(\frac{1}{2} \vee 1-\frac{\alpha}{2} \vee \frac{\alpha-d}{2}\right) .
$$

Hence this scheme meets the outer bound for the rest of the cases.

If we relax the assumption of the symmetric channel and symmetric rate-diversity requirements, the analysis is no longer simple. Our guess is that for this case, the two-

split Han-Kobayashi scheme is no longer optimal. We may need to use generalized schemes with more than two-splits and also obtain better outer bounds.

\section{A Proof of Lemma 1}

Proof of part (i): If $E_{i}^{\epsilon} \subset \hat{\mathcal{O}}_{i}^{c}$, where

$$
E_{i}^{\epsilon}=\left[h^{\prime}, h^{\prime}+\epsilon\right) \times\left[g^{\prime}, g^{\prime}+\epsilon\right),
$$

then $\kappa_{\hat{h}} h^{\prime}+\kappa_{\hat{g}} g^{\prime} \leq d_{i}-\delta$ for some $\delta>0$. Further

$$
\begin{aligned}
\mathbb{P}\left(E_{i}^{\epsilon}\right) & =\mathbb{P}\left(\left[h^{\prime}, h^{\prime}+\epsilon\right) \times\left[g^{\prime}, g^{\prime}+\epsilon\right)\right) \\
& =\mathbb{P}\left(\left[h^{\prime}, h^{\prime}+\epsilon\right)\right) \mathbb{P}\left(\left[g^{\prime}, g^{\prime}+\epsilon\right)\right) \\
& \doteq \mathrm{SNR}^{-\kappa_{\hat{h}} h^{\prime}} \mathrm{SNR}^{-\kappa_{\hat{g}} g^{\prime}} \\
& =\mathrm{SNR}^{-\left(\kappa_{\hat{h}} h^{\prime}+\kappa_{\hat{g}} g^{\prime}\right)} \\
& \geq \mathrm{SNR}^{-d_{i}+\delta},
\end{aligned}
$$


Proof of part (ii): Note that $\hat{\mathcal{O}}_{i}$ corresponds to the unshaded region in Figure 2. To begin with, observe that the region where $\hat{h}_{i}$ or $\hat{g}_{i}$ are negative do not contribute to the probability in the scale of interest. This follows from the exponential tail property of the distribution function (11). More precisely for any arbitrary small $\epsilon>0$

$$
\left.\mathbb{P}\left(\hat{h}_{i} \leq-\epsilon\right)=\mathbb{P}\left(\left|h_{i}\right|^{2}\right) \geq \mathrm{SNR}^{\epsilon}\right) \doteq 0 .
$$

Similarly $\mathbb{P}\left(\hat{g}_{i} \leq-\epsilon\right) \doteq 0$. Therefore

$$
\mathbb{P}\left(\hat{\mathcal{O}}_{i}\right) \doteq \mathbb{P}\left(\hat{\mathcal{O}}_{i}^{\prime}\right)
$$

where

$$
\begin{aligned}
\hat{\mathcal{O}}_{i}^{\prime} & =\hat{\mathcal{O}}_{i} \cap\left\{\mathbb{R}^{+} \times \mathbb{R}^{+}\right\} \\
& =\left\{\kappa_{h_{i}} \hat{h}_{i}+\kappa_{g_{i}} \hat{g}_{i} \geq d_{i}, \hat{h}_{i} \geq 0, \hat{g}_{i} \geq 0\right\}
\end{aligned}
$$

For $X \in\left\{h_{1}, g_{1}, h_{2}, g_{2}\right\}$ and assuming that the density function $f_{|X|^{2}}(x)$ exists for small enough $x$, from (12) it follows that

$$
f_{|X|^{2}}(x) \doteq x^{\left(\kappa_{X}-1\right)},
$$

where the $\doteq$ is in the limit as $x \rightarrow 0$. If $\hat{X}$ is the change of variables according to (17), then it can easily be shown that

$$
f_{\hat{X}}(x) \doteq \mathrm{SNR}^{-\kappa_{\hat{X}} x}, \quad \forall x \geq 0 .
$$

If we let $I=[0, \gamma)^{2}$, where $\gamma>\left(d_{i} / \kappa_{h_{i}} \vee d_{i} / \kappa_{g_{i}}\right)$. Then

$$
\begin{aligned}
\mathbb{P}\left(\hat{\mathcal{O}}_{i}^{\prime}\right) & =\mathbb{P}\left(\hat{\mathcal{O}}_{i}^{\prime} \cap I\right)+\mathbb{P}\left(I^{c}\right) \\
& <\int_{\hat{\mathcal{O}}_{i}^{\prime} \cap I} f_{\hat{h}_{i}}(x) f_{\hat{g}_{i}}(y) d x d y+\mathbb{P}([\gamma, \infty] \times[0, \infty])+\mathbb{P}([0, \infty] \times[\gamma, \infty]) \\
& \doteq \int_{\hat{\mathcal{O}}_{i}^{\prime} \cap I} \mathrm{SNR}^{-\left(\kappa_{\hat{h}_{i}} x+\kappa_{\hat{g}_{i}} y\right)} d x d y+\mathrm{SNR}^{-\gamma \kappa_{\hat{h}_{i}}}+\mathrm{SNR}^{-\gamma \kappa_{\hat{g}_{i}}} \\
& <\gamma^{2} \mathrm{SNR}^{-d_{i}}+\mathrm{SNR}^{-\gamma \kappa_{\hat{h}_{i}}}+\mathrm{SNR}^{-\gamma \kappa_{\hat{g}_{i}}} \\
& \doteq \mathrm{SNR}^{-d_{i}} .
\end{aligned}
$$

Therefore,

$$
\mathbb{P}\left(\hat{\mathcal{O}}_{i}\right) \doteq \mathbb{P}\left(\hat{\mathcal{O}}_{i}^{\prime}\right) \leq \mathrm{SNR}^{-d_{i}}
$$

Since $\left\{\left[d_{i}, \infty\right] \times[0, \infty]\right\} \subset \hat{\mathcal{O}}_{i}^{\prime} \subset$ we have,

$$
\mathbb{P}\left(\hat{\mathcal{O}}_{i}^{\prime}\right) \geq \mathrm{SNR}^{-d_{i}}
$$

Thus we conclude

$$
\mathbb{P}\left(\hat{\mathcal{O}}_{i}\right) \doteq \mathrm{SNR}^{-d_{i}}
$$




\section{B DMT achieved by the Han-Kobayashi scheme}

We characterize the performance of the Han-Kobayashi scheme for the symmetric DMT by characterizing its performance over the compound channel given by the no-outage set. The no-outage set for a symmetric diversity gain of $d$ is given by

$$
\hat{\mathcal{A}}=\left\{\hat{h}_{1}+\hat{g}_{1} \leq d\right\} \times\left\{\hat{h}_{2}+\hat{g}_{2} \leq d\right\}
$$

where

$$
\left(\left|h_{1}\right|^{2},\left|g_{1}\right|^{2},\left|h_{2}\right|^{2},\left|g_{2}\right|^{2}\right)=\left(\mathrm{SNR}^{-\hat{h}_{1}}, \mathrm{SNR}^{-\hat{g}_{1}}, \mathrm{SNR}^{-\hat{h}_{2}}, \mathrm{SNR}^{-\hat{g}_{2}}\right)
$$

We use a simple version of the Han-Kobayashi scheme that was shown to achieve the symmetric capacity within 1 bit per symbol of the interference channel in [1]. For a given block length $n$ transmitter $i$ chooses a private message from codebook $\mathcal{C}_{i, n}^{v}$ and a public message from the codebook $\mathcal{C}_{i, n}^{u}$. The codebooks are generated by using i.i.d. random Gaussian variables with variances $\rho_{v_{i}}=\mathrm{SNR}^{-v_{i}}$ and $\rho_{u_{i}}=1-\rho_{v_{i}}$ respectively. The codebooks have rate $s_{i} \log \mathrm{SNR}$ and $t_{i} \log \mathrm{SNR}$ respectively. After selecting the corresponding codewords user $i$ transmits the signal $\mathbf{x}_{i}=P_{i}\left(\mathbf{c}_{i}^{u}+\mathbf{c}_{i}^{v}\right)$, where $P_{i}$ is the power constraint at the transmitter.

Each receiver performs joint decoding of both the messages from its transmitter and the public message from the other transmitter. The decoding of the private message at receiver 1 is successful if,

$$
\begin{aligned}
s_{1} \log \mathrm{SNR} & \leq \log \left(1+\frac{\rho_{v_{1}}\left|h_{1}\right|^{2} \mathrm{SNR}}{1+\rho_{v_{2}}\left|g_{1}\right|^{2} \mathrm{SNR}^{\alpha}}\right) \\
& \doteq\left(1-\hat{h}_{1}-v_{1}-\left(\alpha-v_{2}-\hat{g}_{1}\right)^{+}\right)^{+} \log \mathrm{SNR} .
\end{aligned}
$$

Therefore, we can write

$$
s_{1} \leq\left(1-\hat{h}_{1}-v_{1}-\left(\alpha-v_{2}-\hat{g}_{1}\right)^{+}\right)^{+}
$$

Note that since the channel state can take any value from the no-outage set, we need to take the minimum over $\hat{\mathcal{A}}$. Therefore

$$
s_{1} \leq \min _{\hat{\mathcal{A}}}\left(1-\hat{h}_{1}-v_{1}-\left(\alpha-v_{2}-\hat{g}_{1}\right)^{+}\right)^{+} .
$$

Similarly, we obtain the other constraints on the sum of sub-rates by considering the decoding at receivers 1 and 2. Analogous to the analysis in [8], we can show that the 
following constraints are the only ones that matter:

$$
\begin{aligned}
s_{1} & \leq a_{11} \stackrel{\text { def }}{=} \min _{\hat{\mathcal{A}}}\left(1-\hat{h}_{1}-v_{1}-\left(\alpha-v_{2}-\hat{g}_{1}\right)^{+}\right)^{+} \\
t_{1}+s_{1} & \leq a_{12} \stackrel{\text { def }}{=} \min _{\hat{\mathcal{A}}}\left(1-\hat{h}_{1}-\left(\alpha-v_{2}-\hat{g}_{1}\right)^{+}\right)^{+} \\
t_{2}+s_{1} & \leq a_{13} \stackrel{\text { def }}{=} \min _{\hat{\mathcal{A}}}\left(\left(1-\hat{h}_{1}-v_{1} \vee \alpha-\hat{g}_{1}\right)-\left(\alpha-v_{2}-\hat{g}_{1}\right)^{+}\right)^{+} \\
t_{2}+t_{1}+s_{1} & \leq a_{14} \stackrel{\text { def }}{=} \min _{\hat{\mathcal{A}}}\left(\left(1-\hat{h}_{1} \vee \alpha-\hat{g}_{1}\right)-\left(\alpha-v_{2}-\hat{g}_{1}\right)^{+}\right)^{+} . \\
s_{2} & \leq a_{21} \stackrel{\text { def }}{=} \min _{\hat{\mathcal{A}}}\left(1-\hat{h}_{2}-v_{2}-\left(\alpha-v_{1}-\hat{g}_{2}\right)^{+}\right)^{+} \\
t_{2}+s_{2} & \leq a_{22} \stackrel{\text { def }}{=} \min _{\hat{\mathcal{A}}}\left(1-\hat{h}_{2}-\left(\alpha-v_{1}-\hat{g}_{2}\right)^{+}\right)^{+} \\
t_{1}+s_{2} & \leq a_{23} \stackrel{\text { def }}{=} \min _{\hat{\mathcal{A}}}\left(\left(1-\hat{h}_{2}-v_{2} \vee \alpha-\hat{g}_{2}\right)-\left(\alpha-v_{1}-\hat{g}_{2}\right)^{+}\right)^{+} \\
t_{1}+t_{2}+s_{2} & \leq a_{24} \stackrel{\text { def }}{=} \min _{\hat{\mathcal{A}}}\left(\left(1-\hat{h}_{2} \vee \alpha-\hat{g}_{2}\right)-\left(\alpha-v_{1}-\hat{g}_{2}\right)^{+}\right)^{+} .
\end{aligned}
$$

The multiplexing gain achieved by each user is given by

$$
r_{i}=s_{i}+t_{i}, \quad i=1,2 .
$$

The symmetric multiplexing gains achievable with the power split $\left(v_{1}, v_{2}\right)$ is then given by

$$
r\left(v_{1}, v_{2}\right)=s_{i}+t_{i}, \quad i=1,2 .
$$

By doing the the Fourier-Motzkin elimination (as in [8]) it can be shown that,

$$
\begin{gathered}
r\left(v_{1}, v_{2}\right)=\min \left\{a_{12}, a_{22},\left(a_{11}+a_{24}\right) / 2,\left(a_{21}+a_{14}\right) / 2,\left(a_{13}+a_{23}\right) / 2,\right. \\
\left.\left(a_{11}+a_{14}+a_{23}\right) / 3,\left(a_{21}+a_{24}+a_{13}\right) / 3\right\} .
\end{gathered}
$$

Note that the non-negativity constraints on the sub-rates need not be taken into consideration for the Fourier-Motzkin elimination (see [5],Section 6). The power-split must be chosen so as to maximize the symmetric rate. Therefore

$$
r_{\mathrm{HK}}(\alpha, d)=\max _{\left(v_{1}, v_{2}\right)} r\left(v_{1}, v_{2}\right) .
$$

This is a non-convex optimization problem. It can be shown that when $v_{1}=v_{2}=v$, the fundamental solution is as shown in Table 1. (Simulations suggest that letting $v_{1} \neq v_{2}$ does not increase the symmetric rate any further.) 


\section{Worst Case Outer Bound for DMT}

We get an outer bound to the DMT by assuming that the transmitter knows the channel coefficients $\left(\hat{h}_{1}, \hat{g}_{1}, \hat{h}_{2}, \hat{g}_{2}\right)$. The transmitters can therefore adopt the power split and the rate split accordingly. We have already mentioned that the power splits must now be chosen to be

$$
v_{1}=\alpha-\hat{g}_{2}, \quad v_{2}=\alpha-\hat{g}_{1} .
$$

Similar to (70)-(77), we obtain the constraint on the sub-rates as

$$
\begin{aligned}
s_{1} & \leq \bar{a}_{11} \stackrel{\text { def }}{=}\left(1-\alpha-\hat{h}_{1}+\hat{g}_{2}\right)^{+} \\
t_{1}+s_{1} & \leq \bar{a}_{12} \stackrel{\text { def }}{=}\left(1-\hat{h}_{1}\right)^{+} \\
t_{2}+s_{1} & \leq \bar{a}_{13} \stackrel{\text { def }}{=}\left(\left(1-\alpha-\hat{h}_{1}+\hat{g}_{2} \vee \alpha-\hat{g}_{1}\right)\right)^{+} \\
t_{2}+t_{1}+s_{1} & \leq \bar{a}_{14} \stackrel{\text { def }}{=}\left(\left(1-\hat{h}_{1} \vee \alpha-\hat{g}_{1}\right)\right)^{+} \\
s_{2} & \leq \bar{a}_{21} \stackrel{\text { def }}{=}\left(1-\alpha-\hat{h}_{2}+\hat{g}_{1}\right)^{+} \\
t_{2}+s_{2} & \leq \bar{a}_{22} \stackrel{\text { def }}{=}\left(1-\hat{h}_{2}\right)^{+} \\
t_{1}+s_{2} & \leq \bar{a}_{23} \stackrel{\text { def }}{=}\left(\left(1-\alpha-\hat{h}_{2}+\hat{g}_{1} \vee \alpha-\hat{g}_{2}\right)\right)^{+} \\
t_{1}+t_{2}+s_{2} & \leq \bar{a}_{24} \stackrel{\text { def }}{=}\left(\left(1-\hat{h}_{2} \vee \alpha-\hat{g}_{2}\right)\right)^{+}
\end{aligned}
$$

Similar to (80) the symmetric rate for a given value of the channel gains is given by,

$$
\begin{gathered}
r\left(\hat{h}_{1}, \hat{g}_{1}, \hat{h}_{2}, \hat{g}_{2}\right)=\min \left\{\bar{a}_{12}, \bar{a}_{22},\left(\bar{a}_{11}+\bar{a}_{24}\right) / 2,\left(\bar{a}_{21}+\bar{a}_{14}\right) / 2,\left(\bar{a}_{13}+\bar{a}_{23}\right) / 2,\right. \\
\left.\left(\bar{a}_{11}+\bar{a}_{14}+\bar{a}_{23}\right) / 3,\left(\bar{a}_{21}+\bar{a}_{24}+\bar{a}_{13}\right) / 3\right\}
\end{gathered}
$$

This quantity is now minimized over the set $\hat{\mathcal{A}}$ to obtain an outer bound to the DMT:

$$
\hat{r}_{\text {out }}(\alpha, d)=\min _{\hat{\mathcal{A}}} r\left(\hat{h}_{1}, \hat{g}_{1}, \hat{h}_{2}, \hat{g}_{2}\right) \text {. }
$$

It can be shown that

$$
\hat{r}_{\text {out }}(\alpha, d)=(1-d) \wedge\left(1-\frac{\alpha}{2}-d \vee \frac{1-d+\alpha}{4}\right) \wedge\left(\alpha-d \wedge 1-\alpha-d \wedge \frac{1+\alpha-d}{3}\right) .
$$

Observe that the outer bound obtained here is tighter than the outer bounds given in [6]. 


\section{Proof of Theorem 3}

We follow an analysis similar to that in Appendix B to characterize the DMT region achieved by the Han-Kobayashi scheme. We will derive inner and outer bounds for the general case with $\beta_{1}, \beta_{2}, \alpha_{1}$ and for diversity $d_{1}$ and $d_{2}$, and then compare the bounds for the special case. We need to find the g.d.o.f. region of the compound channel defined by the no-outage set

$$
\hat{\mathcal{A}}=\left\{\hat{h}_{1}+\hat{g}_{1} \leq d_{1}\right\} \times\left\{\hat{h}_{2} \leq d_{2}\right\},
$$

For transmitter 1 there is no public message, i.e. $v_{1}=0$. Its rate is given by $r_{1} \log \mathrm{SNR}$. For transmitter 2 we use power split $v_{2}=\alpha_{1}$ with multiplexing gains of the private and public messages to be $s_{2} \log$ SNR and $t_{2} \log$ SNR respectively, such that

$$
r_{2}=s_{2}+t_{2}
$$

As earlier, from the decodability constraints at the two receivers, we have

$$
\begin{gathered}
r_{1} \leq \min _{\hat{\mathcal{A}}}\left(\beta_{1}-\hat{h}_{1}\right)^{+}=\left(\beta_{1}-d_{1}\right)^{+} \\
t_{2}+r_{1} \leq \min _{\hat{\mathcal{A}}}\left(\beta_{1}-\hat{h}_{1} \vee \alpha_{1}-\hat{g}_{1}\right)^{+}=\left(\beta_{1}-d_{1} \vee \alpha_{1}-d_{1} \vee \frac{\beta_{1}-d_{1}+\alpha_{1}}{2}\right)^{+} . \\
s_{2} \leq \min _{\hat{\mathcal{A}}}\left(\beta_{2}-\hat{h}_{2}-\alpha_{1}\right)^{+}=\left(\beta_{2}-\alpha_{1}-d_{2}\right)^{+} \\
t_{2}+s_{2} \leq \min _{\hat{\mathcal{A}}}\left(\beta_{2}-\hat{h}_{2}\right)^{+}=\left(\beta_{2}-d_{2}\right)^{+} .
\end{gathered}
$$

By Fourier-Motzkin elimination, the achievable g.d.o.f. with the given Han-Kobayashi scheme is given by

$$
\begin{aligned}
r_{1} & \leq\left(\beta_{1}-d_{1}\right)^{+} \\
r_{2} & \leq\left(\beta_{2}-d_{2}\right)^{+} \\
r_{1}+r_{2} & \leq\left(\beta_{1}-d_{1} \vee \alpha_{1}-d_{1} \vee \frac{\beta_{1}-d_{1}+\alpha_{1}}{2}\right)^{+}+\left(\beta_{2}-\alpha_{1}-d_{2}\right)^{+} .
\end{aligned}
$$

We next consider the worst case outer bound. When the fading level is $\hat{a}=\left(\hat{h}_{1}, \hat{h}_{2}, \hat{g}_{1}\right) \in$ $\hat{\mathcal{A}}$, the g.d.o.f. region for the Z-interference channel $\mathcal{R}(\hat{a})$ is given by

$$
\begin{aligned}
r_{1} & \leq\left(\beta_{1}-\hat{h}_{1}\right)^{+} \\
r_{2} & \leq\left(\beta_{2}-\hat{h}_{2}\right)^{+} \\
r_{1}+r_{2} & \leq\left(\beta_{1}-\hat{h}_{1} \vee \alpha_{1}-\hat{g}_{1}\right)^{+}+\left(\beta_{2}-\alpha_{1}-\hat{h}_{2}+\hat{g}_{1}\right)^{+} .
\end{aligned}
$$


The worst case outer bound is then given by

$$
\begin{aligned}
& \mathcal{R}_{o}\left(d_{1}, d_{2}\right)=\bigcap_{\hat{\mathcal{A}}} \mathcal{R}(\hat{a}) \\
& =\left\{r_{1} \leq \min _{\hat{\mathcal{A}}}\left(\beta_{1}-\hat{h}_{1}\right)^{+}=\left(\beta_{1}-d_{1}\right)^{+},\right. \\
& r_{2} \leq \min _{\hat{\mathcal{A}}}\left(\beta_{2}-\hat{h}_{2}\right)^{+}=\left(\beta_{1}-d_{1}\right)^{+}, \\
& \left.r_{1}+r_{2} \leq \min _{\hat{\mathcal{A}}}\left(\beta_{1}-\hat{h}_{1} \vee \alpha_{1}-\hat{g}_{1}\right)^{+}+\left(\beta_{2}-\alpha_{1}-\hat{h}_{2}\right)^{+}=K\right\},
\end{aligned}
$$

where

$$
K=\left(\beta_{2}-d_{2} \vee \frac{\beta_{1}-\alpha_{1}-d_{1}}{2} \vee \beta_{1}-d_{1} \vee \beta_{1}+\beta_{2}-\alpha_{1}-d_{1}-d_{2} \vee \alpha_{1}-d_{1}\right)^{+} .
$$

It is easy to verify that when $d_{1}=d_{2}=d$ and $\beta_{1}=\beta_{2}=1$, the worst case outer bound meets the inner bound

\section{Acknowledgments}

The authors would like to thank Vaneet Aggarwal for pointing out errors in an earlier version of this paper.

\section{References}

[1] R. H. Etkin, D. Tse, and H. Wang, "Gaussian interference channel capacity to within one bit," IEEE Trans. Inform. Theory, Vol. 54, No. 12, Dec. 2008.

[2] L. Zheng, and D. Tse, "Diversity and multiplexing: a fundamental tradeoff in multiple-antenna channels" IEEE Trans. Inform. Theory, Vol. 49, No. 5, May 2003.

[3] D. Tse, P. Viswanath, and L. Zheng, Diversity-multiplexing tradeoff in multipleaccess channels IEEE Trans. Inform. Theory, Vol. 50, No. 9, Sept. 2004.

[4] S. Tavildar, and P. Viswanath, Approximately universal codes over slow-fading channels IEEE Trans. Inform. Theory, Vol. 52, No. 7, July. 2006.

[5] A. Raja, V. Prabhakaran, and P. Viswanath, "The two-user Gaussian compound interference channel," submitted to the IEEE Trans. Inform. Theory, 2008.

[6] E. Akuiyibo, and O. Leveque, Diversity-multiplexing tradeoff for the slow fading interference channel Intl. Zurich Seminar on Communications (IZS), Mar. 12-14, 2008. 
[7] C. Akçaba, and H. Bölcskei, "On the achievable diversity-multiplexing tradeoff in interference channels," http://arxiv.org/abs/0903.2226.

[8] H. F. Chong, M. Motani, H. K. Garg, and H. E. Gamal, "On the Han-Kobayashi region for the interference channel," IEEE Trans. Inform. Theory, Vol. 54(7), pp. 31883195 , July 2008.

[9] D. Tse and P. Viswanath "Fundamentals of Wireless Communication," Cambridge University Press, May 2005. 